\title{
A NOTE ON GROUPS WITHOUT ISOMORPHIC SUBGROUPS
}

\section{IRVING KAPLANSKY}

1. Introduction. One of the theorems obtained by R. A. Beaumont in a recent paper ${ }^{1}$ states that if $G$ is an abelian group of finite rank all of whose elements have finite order, then $G$ has no proper isomorphic subgroups. If we interpret $G$ as a vector space over the ring of integers $I$, it is natural to raise the question: what properties of $I$ are needed for this result? In this note we find two conditions which are sufficient, given as (1) and (2) in our main theorem. Whether these conditions are also necessary remains to be determined.

2. A preliminary lemma. We shall require the following lemma, which is closely related to various known results. ${ }^{2}$

Lemma. Let $G$ be a vector space over a commutative principal ideal ring $R$. Suppose that $G$ can be spanned by $r$ elements, and that $H$ is a subspace that can be spanned by a finite number of elements of $H$. Then $H$ can be spanned by $r$ elements of $H$.

Proof. Let $G$ be spanned by $g_{1}, \cdots, g_{r} ; H$ by $h_{1}, \cdots, h_{s}$. Then $h_{i}=\sum_{j} \alpha_{i j} g_{j}\left(\alpha_{i j} \in R\right)$. Let $\beta$ be the H.C.F. of $\alpha_{11}, \cdots, \alpha_{s 1}$, so that $\beta=\sum_{i} \lambda_{i} \alpha_{i 1}$. Define $k_{1}=\sum_{i} \lambda_{i} h_{i}$. Then $k_{1} \in H$, and since

$$
k_{1}=\beta g_{1}+\text { a linear combination of } g_{2}, \cdots, g_{r} \text {, }
$$

$k_{1}, g_{2}, \cdots, g_{r}$ span $H$. After $r$ such steps we shall obtain elements $k_{1}, \cdots, k_{r}$ in $H$ which span $H$.

3. The theorem. Let $V$ be a vector space over a ring $R$.

Definition. $V$ has rank $r$ over $R$ if any finite subset of $V$ can be spanned over $R$ by $r$ elements of $V$, and if $r$ is the smallest integer with this property.

THEOREM. Let $V$ have finite rank $r$ over $R$ and suppose that:

(1) $R$ is a commutative principal ideal ring.

(2) Every proper residue class ring of $R$ is finite.

(3) For every $v \in V$, there is an $\alpha \neq 0$ in $R$ such that $\alpha v=0$.

Then $V$ has no isomorphic proper subspaces.

Presented to the Society, April 28, 1945; received by the editors March 12, 1945.

1 Groups with isomorphic proper subgroups, Bull. Amer. Math. Soc. vol. 51 (1945) pp. 381-387.

2 For example, C. J. Everett, Vector spaces over rings, Bull. Amer. Math. Soc. vol. 48 (1942) pp. 312-316. 
Proof. Take any $v \in V$ and an $\alpha \neq 0$ with $\alpha v=0$. Let $W$ be the subspace consisting of all $w$ with $\alpha w=0$, and let $A$ be the ideal in $R$ consisting of all $\beta$ with $\beta W=0$. By hypothesis $R / A$ is finite; say it has $n$ elements. Then $W$ is finite; in fact, it has at most $n^{r}$ elements. For take any $k$ elements of $W$ and let $H$ be the subspace they span. By our hypothesis $H$ can be embedded in a subspace $G$ spanned by $r$ elements. Then by our lemma, $H$ can also be spanned by $r$ elements $h_{1}, \cdots, h_{r}$ of $H$. Since $H$ is annihilated by $A$, there are at most $n^{r}$ different elements obtainable by taking linear combinations of $h_{1}, \cdots, h_{r}$. Hence $k \leqq n^{r}$, as desired.

Now suppose there is an isomorphism mapping $V$ into a proper subspace $V^{\prime}$. In this mapping $W$ must be sent into part of itself, for $W$ consists of all elements annihilated by $\alpha$. But $W$ is finite; hence $W$ must be mapped into all of itself. In particular, the (arbitrary) element $v$ is part of the image space $V^{\prime}$, whence $V^{\prime}=V$.

New York City 